\title{
On the Mathematical Description of Quantized Fields
}

\author{
R. AsColi *, G. EPIFANio, and A. Restivo \\ Istituto di Fisica dell'Università - Palermo \\ Istituto Nazionale di Fisica Nucleare - Sezione Siciliana
}

Received June 6, 1969, in revised form March 26, 1970

\begin{abstract}
We start from Haag's proposal to describe quantum fields at a point, corresponding to the heuristic description by means of their matrix elements $(A(x) \Phi \mid \Psi)$ between vectors of a dense linear manifold $D$ of the Hilbert space. We particularize this idea, so that the sesquilinear functional that describes the field at a point may be considered as an element of the sequential completion of a space of operators, endowed with a suitable " $D$-weak" topology.

It is shown that any Wightman field may be described in this way, as a rather elementary consequence of the existence of a translation invariant cyclic vacuum. Furthermore the field turns out to be an infinitely differentiable function of space and time.
\end{abstract}

\section{§ 1. Introduction}

It has been first proposed by Haag (1963, Ref. [1]) that the quantum field $A(x)$ at any point $x$ should be described by means of a sesquilinear functional over a dense linear manifold $D$ of the Hilbert space $H$, corresponding to the heuristically defined mapping $(\Phi, \Psi) \rightarrow(A(x) \Phi \mid \Psi)$.

The work of Kristensen, Mejlbo, and Poulsen (1965, Ref. [2]) and Grossmann (1967, Ref. [3]) may be considered as providing two different topological particularizations of this idea. These authors show, among other things, that free fields and Wick products of them may be described, in their sense, as infinitely differentiable functions of space and time.

In this work we consider another topological particularization of Haag's proposal, which is very simple, and we show that essentially any Wightman field may be described in this way as an infinitely differentiable function of space and time. We only require the existence of a cyclic translation-invariant vacuum.

\section{$\S 2 . C_{D}$ : A non Commutative Topological $*$-Algebra of Unbounded Operators}

Definition 1. We use the following notation: $R$ means the real field, $M$ the (Minkowski) space-time, which in this paper may be identified with the m-dimensional real space $R^{m}$ (where the physically most interesting value of $m$ is 4), $C$ means the complex field.

\footnotetext{
$\star$ Now at Istituto di Fisica dell'Università - Lecce.
} 
For any Hausdorff space $X$ we call $\tilde{X}$ its sequential completion and $\hat{X}$ its completion.

We say that a function on $M$ is $\mathscr{C}^{\infty}$ if it is infinitely differentiable. We call $\mathscr{C}^{\infty}\left(M^{n}\right)$ the space of the complex valued $\mathscr{C}^{\infty}$ functions on $M^{n}$ and $\mathscr{D}\left(M^{n}\right)$ its linear manifold of the functions with compact support, endowed with their usual topologies. We call $\mathscr{D}^{\prime}\left(M^{n}\right)$ the space of the distributions over $M^{n}$, endowed with its usual topology.

For any distribution $\mathscr{A}$ and for any test-function $f$ we write also $\int \mathscr{A}(x) f(x) d x$ instead of $\mathscr{A}(f)$.

We are especially concerned with the space $C_{D}$ defined as follows.

Definition 2. Let $D$ be a scalar product space. We say that any linear operator $A$ defined on $D$ has an adjoint $A^{*}$ in $D$ whenever there exists a linear operator $A^{*}$ defined on $D$ such that:

$$
\forall \Phi, \Psi \in D \quad(A \Phi \mid \Psi)=\left(\Phi \mid A^{*} \Psi\right) .
$$

We call $C_{D}$ the set of the linear operators that are defined on $D$ and have an adjoint in $D$, endowed by the $D$-weak topology, determined by the set of seminorms $\{A \rightarrow \mid(A \Phi \mid \Psi) \| \Phi, \Psi \in D\}$.

We call $B_{D}$ the topological linear submanifold of $C_{D}$ consisting of the bounded operators.

Let us restate the definition of $C_{D}$ in a perhaps more familiar language.

Let $H$ be a Hilbert space and $D$ a dense linear manifold of $H$. Let $W_{D}$ be the set of the linear operators of $H$ such that: their domain contains $D$, the domain of their adjoints contains $D$ and $D$ is left invariant by them and by their adjoints. That is $W_{D}$ is the set of the operators that satisfy the requirements that are usually imposed to the range of a Wightman field. Let us endow $W_{D}$ with the " $D$-weak" topology. Then $C_{D}$ is the quotient structure of $W_{D}$ with respect to the equivalence relation that expresses the equality of the restrictions to $D$ of any two operators.

The Definition 2 of $C_{D}$ appears to be the appropriate one for the space where a Wightman field takes its values, in order to consider the Wightman field as a vector-valued distribution: in fact this choice allows us to overcome the difficulties, that the addition of operators with different domains does not have the group property and that $W_{D}$, with the appropriate topology, which is just the " $D$-weak" topology, is not a Hausdorff space.

Theorem 1. For any scalar product space $D, C_{D}$ (endowed with the natural operations) is a topological *-algebra in the following sense:

a) $C_{D}$ is an algebra with involution,

b) $C_{D}$ is a locally convex Hausdorff space,

c) the product is separately continuous,

d) the involution is continuous.

$B_{D}$ is a topological $*$-subalgebra of $C_{D}$. 
Proof. The proof is straightforward.

We first remark that for any $A \in C_{D}$ Definition 2 determines uniquely an $A^{*} \in C_{D}$ which is adjoint to $A$ in $D$.

Let $A, B \in C_{D}$. As $A, B, A^{*}, B^{*}$ are defined on the whole $D$, also $A+B, A^{*}+B^{*}, A B, B^{*} A^{*}$ are defined on the whole $D$ and it is trivial to check that $A^{*}+B^{*}$ is adjoint in $D$ to $A+B$ and $B^{*} A^{*}$ is adjoint in $D$ of $A B$. So it follows firstly that $A+B \in C_{D}$ and $A B \in C_{D}$ and secondly that all the algebraic requirements are satisfied.

$C_{D}$ is an Hausdorff space because $\forall \Phi, \Psi \in D|(A \Phi \mid \Psi)|=0$ implies $\forall \Phi \in D A \Phi=0$, that is $A=0$.

The separate continuity of the product and the continuity of the involution arise just in the same way as they arise when considering the algebra of the bounded operators of an Hilbert space, endowed with the weak topology: concerning the continuity of the mapping $A \rightarrow A B$, for any $\Phi, \Psi \in D$ requiring $A$ to be such that $|(A B \Phi \mid \Psi)|<\varepsilon$ is just to ask that $A$ be in a $D$-weak neighbourhood of 0 ; concerning the continuity of the mapping $A \rightarrow B A$ one proceeds analogously remarking that $|(B A \Phi \mid \Psi)|<\varepsilon$ if and only if $\left|\left(A \Phi \mid B^{*} \Psi\right)\right|<\varepsilon$, etc.

So all the statements concerning $C_{D}$ are satisfied. The final statement concerning $B_{D}$ follows trivially.

\section{§3. The Completion of $C_{D}$}

In the following we are interested to the sequential completion $\tilde{C}_{D}$ of $C_{D}$.

We remark firstly that the product that is defined within $C_{D}$ allows us to define by continuous extension a left and a right product of any element of the completion $\hat{C}_{D}$ of $C_{D}$ with any element of $C_{D}$, according to the next definition.

Definition 3. Let $D$ be a scalar product space and let $\hat{C}_{D}$ be the completion of $C_{D}$, considered as a Hausdorff vector space.

We call the product $(A, B) \rightarrow A B$ the mapping from $\hat{C}_{D} \times C_{D}$ into $\hat{C}_{D}$ that for any $B \in C_{D}$ extends continuously the mapping $A \rightarrow A B$ of $C_{D}$ into itself. Analogously we define a product from $C_{D} \times \hat{C}_{D}$ into $\hat{C}_{D}$.

We call the involution $A \rightarrow A^{*}$ on $\hat{C}_{D}$ the continuous extension of the involution on $C_{D}$.

We say that an element $A$ of $\hat{C}_{D}$ is Hermitian if $A^{*}=A$.

As a consequence of the principle of conservation of identities the product we have defined is bilinear and the involution antilinear.

Starting from the fact that any operator $A$ of $C_{D}$ may be described by the sesquilinear functional $F_{A}$ on $D:(\Phi, \Psi) \rightarrow F_{A}(\Phi, \Psi)=(A \Phi \mid \Psi)_{2}$ it is easily seen that even any element of the sequential completion $\widetilde{C}_{D}$ of 
$C_{D}$ may correspondingly be described by a sesquilinear functional on $D$. Actually this description is even possible for all the elements of the completion $\hat{C}_{D}$ of $C_{D}$ and it turns out that in this way all the sesquilinear functionals are obtained. These statements are expressed more precisely in the next propositions.

Definition 4. Let $D$ be a scalar product space. We call $S_{D}$ the space of the sesquilinear functionals on $D$, endowed with the topology of pointwise convergence, which is determined by the set of seminorms $\{F \rightarrow|F(\Phi, \Psi)| \mid \Phi, \Psi \in D\}$.

$S_{D}$ is clearly a complete locally convex Hausdorff space.

Theorem 2. Let $D$ be a scalar product space. The mapping from $C_{D}$ into $S_{D}$ defined for any $A \in C_{D}$ by $A \rightarrow F_{A}$, where

$$
\forall \Phi, \Psi \in D \quad F_{A}(\Phi, \Psi)=(A \Phi \mid \Psi)
$$

is one-to-one and bicontinuous. Its continuous extension to $\hat{C}_{D}$ provides an isomorphism of the topological vector spaces $\hat{C}_{D}$ and $S_{D}$. We still write it $A \rightarrow F_{A}$.

Further $\hat{B}_{D}=\hat{C}_{D}$.

This theorem is an easy consequence of known facts concerning weak topologies ${ }^{1}$. The statement that the extended mapping $A \rightarrow F_{A}$ is one to one and bicontinuous is evident and it is the only one we need.

Let us remark explicitly some easy consequences which are used in the following.

Corollary 1. Let $D$ be a scalar product space.

a) Let $\left(A_{n}\right)$ be a sequence in $\hat{C}_{D}$ and $A \in \hat{C}_{D}$. Then $\lim _{n \rightarrow \infty} A_{n}=A$ if and only if $\forall \Phi, \Psi \in D \lim _{n \rightarrow \infty} F_{A_{n}}(\Phi, \Psi)=F_{A}(\Phi, \Psi)$.

b) Let $A$ be a function from $M=R^{m}$ into $\tilde{C}_{D}$. Then $x \rightarrow A(x)$ is infinitely differentiable if and only if $\forall \Phi, \Psi \in D x \rightarrow F_{A(x)}(\Phi, \Psi)$ is infinitely differentiable.

c) Let $A$ be a function from $M=R^{m}$ into $\hat{C}_{D}$ and $B \in \hat{C}_{D}$. Then $x \rightarrow A(x)$ is integrable and $\int A(x) d x=B$ if and only if $\forall \Phi, \Psi \in D \quad x \rightarrow F_{A(x)}(\Phi, \Psi)$ is integrable and ${ }^{2} \int F_{A(x)}(\Phi, \Psi) d x=F_{B}(\Phi, \Psi)$.

We report the following statement, which is not used in the following. The proof shall be published elsewhere.

Theorem 3. For any scalar product space $D$ the sequential completions $\tilde{B}_{D}$ and $\tilde{C}_{D}$ of the topological vector spaces $B_{D}$ and $C_{D}$ respectively coincide.

${ }^{1}$ See Ref. [4] Ch. II, $\S 6, n^{\circ}$ 7, Proposition 9.

${ }^{2}$ This statement may well be considered as a definition of integrable $A$ and $\int A(x) d x=B$. 


\section{§ 4. $\widetilde{C}_{D^{-}}$-valued Fields and Wightman Fields}

Let us now specialize Haag's proposal for the description of quantum fields at a point, according to the next definition.

Definition 5. Let $D$ be a dense linear manifold of an Hilbert space $H$. We call a $\tilde{C}_{D}$ - valued field any mapping $A$ from the (Minkowski) spacetime $M$ into the sequential completion ${ }^{3} \tilde{C}_{D}$ of $C_{D}$ :

$$
A: M \rightarrow \tilde{C}_{D}
$$

The field is called Hermitian if $\forall x \in M A(x)$ is Hermitian (Def. 3).

Concerning such fields we consider here only the following axioms:

Axiom I (Translation invariance). There exists in $H$ a strongly continuous unitary representation $U$ of the group of translations of $M$ such that it leaves $D$ invariant and $\forall x, a \in M U(a) A(x) U(a)^{-1}=A(x+a)$ where $U(a)$ is the representer of the translation $x \rightarrow x+a$ and the products are defined according to Definition 3.

There is clearly no difficulty to formulate in an analogous way the invariance under any group of transformations of $M$, such as the Poincare group.

Axiom II (Existence of a translation invariant vacuum). There exists a vector $\Omega \in D$ such that $\forall a \in M U(a) \Omega=\Omega$.

In order to show that essentially any Wightman field may be described in this way we need to consider the Wightman fields as vectorvalued distributions. According to the remarks following the Definition 2 of $C_{D}$, this is only possible after some particularization of the usual definition of a Wightman field. Essentially the behaviour of the smeared fields outside the common domain $D$ has to be disregarded ${ }^{4}$. We remark first the following immediate consequence of Theorem 2, to guarantee the equivalence of the first and the second parts of the definition to be given.

Lemma 1. Let $D$ be a scalar product space and let $\mathscr{A}$ be a mapping from $\mathscr{D}(M)$ (Def. 1) into $C_{D}$ (Def. 2).

Then $f \rightarrow \mathscr{A}(f)$ is a $C_{D}$ valued distribution ${ }^{5}$ on $M$ (that is $\mathscr{A}$ is continuous) if and only if $\forall \Phi, \Psi \in D f \rightarrow(\mathscr{A}(f) \Phi \mid \Psi)$ is a complex valued distribution over $M$ (that is a continuous mapping from $\mathscr{D}(M)$ into the complex field $C$ ).

\footnotetext{
${ }^{3}$ According to Theorem 3 we are allowed to write $\tilde{B}_{D}$ instead of $\tilde{C}_{D}$.

${ }^{4}$ See Ref. [7] $\S 2.2$.

${ }^{5}$ See Ref. [5] Ch. I, $\S 2$ Définition at page 49.
} 
Definition 6. Let $D$ be a dense linear manifold of an Hilbert space $H$.

We call (scalar) Wightman field over $D$ a mapping $\mathscr{A}$ from $\mathscr{D}(M)$ into $C_{D}$ such that $\forall \Phi, \Psi \in D$ the mapping from $\mathscr{D}(M)$ into the complex field: $f \rightarrow(\mathscr{A}(f) \Phi \mid \Psi)$ is a distribution (that is it is continuous).

Equivalently we call the mapping

$$
\mathscr{A}: \mathscr{D}(M) \rightarrow C_{D}
$$

a Wightman field if it is continuous, that is if it is a $C_{D}$ valued distribution on $M$.

The Wightman field will be called Hermitian if $\forall f \in \mathscr{D}(M) \mathscr{A}^{*}(f)=\mathscr{A}(\bar{f})$.

Concerning Wightman fields we need only the following axioms:

Axiom W I (Translation invariance). There exists in $H$ a strongly continuous unitary representation $U$ of the group of the translations of $M$ such that it leaves $D$ invariant and

$$
\forall a \in M \forall f \in \mathscr{D}(M) U(a) \mathscr{A}(f) U(a)^{-1}=\mathscr{A}\left(f_{a}\right)
$$

where $\forall x \in M f_{a}(x)=f(x-a)$.

Axiom W II (Existence of a translation invariant vacuum). Identical to Axiom II.

Axiom W III (Cyclicity of the vacuum). $\Omega$ is a cyclic vector for the algebra generated by the set of operators $\{\mathscr{A}(f) \mid f \in \mathscr{D}(M)\}$.

Let us recall the following Definition.

Definition 7. Let $M=R^{m}$ and let $X$ be any locally convex Hausdorff space. Let $T$ be an $X$ valued distribution ${ }^{5}$ on $M$.

We say that $T$ " $i s$ " a function if there exists a locally integrable $X$ valued function $F$ on $M$ such that

$$
\forall f \in \mathscr{D}(M) T(f)=\int F(x) f(x) d x
$$

If furthermore $F$ is $\mathscr{C}^{\infty}$ on $M$, we say that $T$ "is" $\mathscr{C}^{\infty}$ on $M$.

Clearly the function $F$ is defined uniquely by the distribution $T$, whenever it is continuous.

Let us now prove the main statement.

Theorem 4. Let $D$ be a dense linear manifold of an Hilbert space $H$ and $\mathscr{A}$ an Hermitian (scalar) Wightman field over $D$ satisfying the Axioms $W I$ (Translation invariance), WII (Existence of a translation invariant vacuum $\Omega \in D$ ) and $W I I I$ (Cyclicity of the vacuum $\Omega$ ).

Let us call $D_{0}$ the linear manifold that is obtained by applying to the vacuum $\Omega$ the algebra generated by the set of operators $\{\mathscr{A}(f) \mid f \in \mathscr{D}(M)\}$. 
Then $\forall \Phi, \Psi \in D_{0}$ the distribution $f \rightarrow(\mathscr{A}(f) \Phi \mid \Psi)$ "is" a $\mathscr{C}^{\infty}(M)$ function $^{6}$, that is explicitly $\forall \Phi, \Psi \in D_{0}$ there exists a $\mathscr{C}^{\infty}(M)$ function $x \rightarrow F_{x}(\Phi, \Psi)$ such that

$$
\forall f \in \mathscr{D}(M)(\mathscr{A}(f) \Phi \mid \Psi)=\int F_{x}(\Phi, \Psi) f(x) d x .
$$

Let us further call $\mathscr{A}_{0}$ the Wightman field over $D_{0}$ defined $\forall f \in \mathscr{D}(M)$ by $\mathscr{A}_{0}(f)=\left.\mathscr{A}(f)\right|_{D_{0}}$.

Then $\mathscr{A}_{0}$, considered as a $\tilde{C}_{D_{0}}$ valued distribution over $M$ :

$$
\mathscr{A}_{0}: \mathscr{D}(M) \rightarrow \tilde{C}_{D_{0}}
$$

"is" a $\mathscr{C}^{\infty}$ function, that is there exists a unique $\mathscr{C}^{\infty} \tilde{C}_{D_{0}}$-valued field $A$ :

such that

$$
A: M \rightarrow \tilde{C}_{D_{0}}
$$

$$
\forall f \in \mathscr{D}(M) \mathscr{A}_{0}(f)=\int A(x) f(x) d x .
$$

At any point $x$ the value $A(x)$ of the field $A$ is described, according to Theorem 2, by the sesquilinear functional over $D_{0}: F_{x}:(\Phi, \Psi) \rightarrow F_{x}(\Phi, \Psi)$, that is, with the notation of Theorem 2, it is $F_{A(x)}=F_{x}$.

The $\tilde{C}_{D_{0}}$-valued field $A$ is Hermitian and it satisfies the Axioms I (Translation invariance) and II (Existence of a translation invariant vacuum) ${ }^{7}$.

Proof. Let us define as usually for any positive integer $n$ the distribution $\mathscr{W}_{n}$ over $M^{n}$ through

$$
\forall f_{1}, \ldots, f_{n} \in \mathscr{D}(M) \mathscr{W}_{n}\left(f_{1} \otimes \ldots f_{n}\right)=\left(\mathscr{A}\left(f_{1}\right) \ldots \mathscr{A}\left(f_{n}\right) \Omega \mid \Omega\right) .
$$

As a consequence of the translation invariance of $\Omega, \mathscr{W}_{n}$ is invariant under the simultaneous translations of all its variables.

As $\Omega$ is cyclic for the algebra generated by the smeared fields, it follows $\forall \Phi, \Psi \in D_{0}$ and $\forall f \in \mathscr{D}(M)$ that $(\mathscr{A}(f) \Phi \mid \Psi)$ is a finite sum of terms of the type $\left(\mathscr{A}(f) \Phi^{\prime} \mid \Psi^{\prime}\right)$, where $\Phi^{\prime}, \Psi^{\prime}$ are such that there exist positive integers $r, n$, with $r \leqq n$ and functions $f_{1}, \ldots, f_{n} \in \mathscr{D}(M)$, so that

$$
\begin{aligned}
\left(\mathscr{A}(f) \Phi^{\prime} \mid \Psi^{\prime}\right) & =\left(\mathscr{A}(f) \mathscr{A}\left(f_{r+1}\right) \ldots \mathscr{A}\left(f_{n}\right) \Omega \mid \mathscr{A}\left(\bar{f}_{r}\right) \ldots \mathscr{A}\left(\bar{f}_{1}\right) \Omega\right) \\
& =\left(\mathscr{A}\left(f_{1}\right) \ldots \mathscr{A}\left(f_{r}\right) \mathscr{A}(f) \mathscr{A}\left(f_{r+1}\right) \ldots \mathscr{A}\left(f_{n}\right) \Omega \mid \Omega\right) \\
& =\mathscr{W}_{n+1}\left(f_{1} \otimes \ldots f_{r} \otimes f \otimes f_{r+1} \otimes \ldots f_{n}\right) .
\end{aligned}
$$

${ }^{6}$ The statement that it "is" a function may be found in Ref. [6], page 872 .

${ }^{7}$ By assuming that $\mathscr{A}$ is a tempered distribution or by using the spectrum condition (see Ref. [7], 2.1, page 35) there would be no difficulty to replace in this theorem $\mathscr{D}(M)$ with $\mathscr{S}(M)$ (see Ref. [8]), so that $D_{0}$ would be enlarged. There is even no difficulty in enlarging further $D_{0}$ to the linear manifold $D_{1}$ that is defined for instance in Ref. [9], 3.3, page 107. This fact appears clearly from the proof.

21 Commun math. Phys., Vol. 18 
This relation may formally be written $\left(\mathscr{A}(x) \Phi^{\prime} \mid \Psi^{\prime}\right)=\int \mathscr{W}_{n+1}\left(x_{1}, \ldots, x_{r}, x, x_{r+1}, \ldots, x_{n}\right) f_{1}\left(x_{1}\right) \ldots f_{n}\left(x_{n}\right) d x_{1} \ldots d x_{n}$.

The distribution $\mathscr{W}_{n+1}$ over $M^{n+1}$, as a consequence of its translation invariance, may be expressed by a distribution $\mathscr{V}_{n}$ over $M^{n}$, such that it may formally be written

$$
\mathscr{W}_{n+1}\left(x_{1}, \ldots, x_{r}, x, x_{r+1}, \ldots, x_{n}\right)=\mathscr{V}_{n}\left(x-x_{1}, \ldots, x-x_{n}\right) .
$$

So we may formally write

$$
\begin{aligned}
\left(\mathscr{A}(x) \Phi^{\prime} \mid \Psi^{\prime}\right) & =\int \mathscr{V}_{n}\left(x-x_{1}, \ldots, x-x_{n}\right) f_{1}\left(x_{1}\right) \ldots f_{n}\left(x_{n}\right) d x_{1} \ldots d x_{n} \\
& =\int \mathscr{V}_{n}\left(\xi_{1}, \ldots, \xi_{n}\right) f_{1}\left(x-\xi_{1}\right) \ldots f_{n}\left(x-\xi_{n}\right) d \xi_{1} \ldots d \xi_{n} .
\end{aligned}
$$

This latter expression represents the convolution of the distribution $\mathscr{V}$ of $\mathscr{D}^{\prime}\left(M^{n}\right)$ with the function $f_{1} \otimes \ldots f_{n}$ of $\mathscr{D}\left(M^{n}\right)$, which is well known to "be" an infinitely differentiable function, the regularized of $\mathscr{V}$ by $f_{1} \otimes \ldots f_{n}$.

So the statement concerning the matrix elements of the field is proved.

Let us now prove the statements concerning the field at a point. Let $\delta_{x}$ be the Dirac $\delta$ at the point $x \in M$. Then it is known that there exists a sequence $\left(f_{x n}\right)$ of functions of $\mathscr{D}(M)$ such that $\delta_{x}=\lim _{n \rightarrow \infty} f_{x n}$, the convergence occurring in the topology of the dual ${ }^{8} \mathscr{E}^{\prime}(M)$ of $\mathscr{C}^{\infty}(M)$.

Let us define $A_{x n}=\mathscr{A}_{0}\left(f_{x n}\right)$. Then it is easily seen that $\left(A_{x n}\right)$ is a Cauchy sequence in $C_{D_{0}}$, or equivalently (Corollary 1 a)) that $\forall \Phi, \Psi \in D_{0}$ the sequence $\left(\left(A_{x n} \Phi \mid \Psi\right)\right)$ is a Cauchy sequence of complex numbers. In fact, using the first part of the theorem we obtain:

$$
\begin{aligned}
\forall x \in M \forall \Phi, \Psi \in D_{0} & F_{x}(\Phi, \Psi)=\int F_{y}(\Phi, \Psi) \delta_{x}(y) d y \\
& =\lim _{n \rightarrow \infty} \int F_{y}(\Phi, \Psi) f_{x n}(y) d y=\lim _{n \rightarrow \infty}\left(A_{x n} \Phi \mid \Psi\right),
\end{aligned}
$$

so that $\forall \Phi, \Psi \in D_{0}$ the sequence $\left(\left(A_{x n} \Phi \mid \Psi\right)\right)$ actually converges to $F_{x}(\Phi, \Psi)$.

As $\forall x\left(A_{x n}\right)$ is a Cauchy sequence in $C_{D_{0}}$, there exists $A(x) \in \tilde{C}_{D_{0}}$ such that $A(x)=\lim _{n \rightarrow \infty} A_{x_{n}}$. Furthermore, according to Corollary $\left.1 \mathrm{a}\right), A(x)$ is described by the sesquilinear functional $F_{x}$, that is $F_{A(x)}=F_{x}$. So $\forall \Phi$, $\Psi \in D_{0}$, according to the fist part of the theorem, it is

$$
\forall f \in \mathscr{D}(M)\left(\mathscr{A}_{0}(f) \Phi \mid \Psi\right)=\int F_{A(x)}(\Phi, \Psi) f(x) d x
$$

so that (Corollary $1 \mathrm{c})$ )

$$
\forall f \in \mathscr{D}(M) \mathscr{A}_{0}(f)=\int A(x) f(x) d x .
$$

${ }^{8}$ See Ref. [8], Ch. III, §3, Théorème XIII. 
As moreover, according to the first part of the theorem, $\forall \Phi, \Psi \in D_{0}$ the function $x \rightarrow F_{A(x)}(\Phi, \Psi)$ is $\mathscr{C}^{\infty}$ over $M$ it follows (Corollary $1 b$ )) that the function $A: x \rightarrow A(x)$ is $\mathscr{C}^{\infty}$ over $M$.

Concerning the Hermiticity of the field we get easily, from the continuity of the involution within $\hat{C}_{D_{0}}$ (Def. 3):

$$
\forall f \in \mathscr{D}(M)\left(\int A(x) f(x) d x\right)^{*}=\int A^{*}(x) \bar{f}(x) d x .
$$

Then, starting from the formula $\forall f \in \mathscr{D}(M) \mathscr{A}_{0}(f)=\int A(x) f(x) d x$, it follows easily that Hermiticity holds for $A$ if and only if it holds for $\mathscr{A}_{0}$.

Analogously, concerning translation invariance, we get from the continuity of the left and right products of any element of $\hat{C}_{D_{0}}$ with any element of $C_{D_{0}}$ (Def. 3):

$$
\begin{aligned}
\forall a \in M \quad \forall f \in \mathscr{D}(M) U_{0}(a) & \left(\int A(x) f(x) d x\right) U_{0}(a)^{-1} \\
& =\int U_{0}(a) A(x) U_{0}(a)^{-1} f(x) d x .
\end{aligned}
$$

Then, starting again from the formula $\forall f \in \mathscr{D}(M) \mathscr{A}_{0}(f)=\int A(x) f(x) d x$, it follows easily that translation invariance holds for $A$ if and only if it holds for $\mathscr{A}_{0}$.

So the proof of Theorem 4 is completed.

The first part of the next proposition provides a rather trivial partial converse of Theorem 4; the second part follows immediately from Theorem 4.

Theorem 5. Let $D$ be a dense linear manifold of an Hilbert space $H$ and $A: M \rightarrow \tilde{C}_{D}$ an Hermitian $\tilde{C}_{D}$-valued field. Let $A$ be locally integrable and

$$
\forall f \in \mathscr{D}(M) \int A(x) f(x) d x \in C_{D} .
$$

Then the mapping $\mathscr{A}$ from $\mathscr{D}(M)$ into $C_{D}$ defined by

$$
\mathscr{A}: f \rightarrow \mathscr{A}(f)=\int A(x) f(x) d x
$$

is an Hermitian Wightman field over D. Further

$$
\forall \Phi, \Psi \in D \forall f \in \mathscr{D}(M)(\mathscr{A}(f) \Phi \mid \Psi)=\int F_{A(x)}(\Phi, \Psi) f(x) d x
$$

where $F_{A(x)}$ is defined according to Theorem 2.

If moreover translation invariance holds for the field $A$, it holds also for the Wightman field $\mathscr{A}$.

Let us furthermore assume that there exists a translation invariant vacuum $\Omega$, such that the linear manifold $D_{0}$ obtained by applying to $\Omega$ the algebra generated by the set of operators $\left\{\int A(x) f(x) d x \mid f \in \mathscr{D}(M)\right\}$ is dense in $H$. 
Then the Hermitian $\tilde{C}_{D_{0}}$-valued field $A_{0}$ over $D_{0}$ defined, according to Theorem 2, by the formula

$$
\forall x \in M \forall \Phi, \Psi \in D_{0} \quad F_{A_{0}(x)}(\Phi \mid \Psi)=F_{A(x)}(\Phi \mid \Psi)
$$

is a $\mathscr{C}^{\infty}$ function on $M$.

Proof. The fact that $\mathscr{A}$ is a $C_{D}$ valued distribution over $M$ follows from Corollary $1 \mathrm{c}$ ). The fact that the field is Hermitian and satisfies translation invariance arises from the last part of the proof of Theorem 4. The remaining statements follow from the application of Theorem 4.

Acknowledgement. The authors wish to thank Dr. Aldo Brigaglia for helpful discussions.

\section{References}

1. Haag, H.: Ann. Phys. 11, 29 (1963).

2. Kristensen, P., Mejlbo, L., Thoue Poulsen, E.: Commun. Math. Phys. 1, 175 (1965).

3. Grossmann, A.: Commun. Math. Phys. 4, 203 (1967).

4. Bourbaki, N.: Eléments de Mathématique: Espaces vectorielles topologiques, $\left(2^{\circ}\right.$ ed.). Ch. I et II. Paris: Hermann 1966.

5. Schwartz, L.: Théorie des Distributions a valeurs vectorielles. Université de Grenoble, Annales de l'Institute Fourier. Chartres: Durand 1959.

6. Schmidt, W., Baumann, K.: Nuovo Cimento 4, 860 (1956).

7. Wightman, A.: Axiomatic field theory. In Theoretical Physics. Vienna: I.A.E.A. 1963.

8. Schwartz, L.: Théorie des Distributions. Tomes I et II. Paris: Hermann 1957 et 1959.

9. Streater, R. F., Wightman, A. S.: PCT, Spin and Statistics, and all that. New YorkAmsterdam: Benjamin 1964.

R. Ascoli

Istituto di Fisica dell'Università

Via P. Giuria 1

I-10125 Torino/Italien

G. Epifanio

Istituto di Fisica dell'Universitá

Via Archirafi 36

90123 Palermo/Italy

A. Restivo

Laboratorio di Cibernetica del C.N.R.

80072 Arco Felice (Napoli)/Italy 